\title{
Co-elevated expression of hepatocyte growth factor and interleukin-8 contributes to poor prognosis of patients with primary nasopharyngeal carcinoma
}

\author{
LI-QIONG XIE ${ }^{1}$, LI-JUAN BIAN ${ }^{2}$, ZHI LI ${ }^{1}$, YANG LI $^{1}$ and YING-JIE LIANG ${ }^{1}$ \\ ${ }^{1}$ Department of Pathology, 1st Affiliated Hospital, ${ }^{2}$ Department of Pathology, \\ 2nd Affiliated Hospital, Sun Yat-sen University, Guangzhou 510080, P.R. China
}

Received August 28, 2009; Accepted October 13, 2009

DOI: $10.3892 /$ or_00000615

\begin{abstract}
Hepatocyte growth factor (HGF) related tumor angiogenesis and prognosis of patients with nasopharyngeal carcinoma (NPC) has not been identified. The expressions of HGF and IL-8, as well as microvessels density were evaluated in 127 NPC biopsies by immunohistochemical staining. The correlation between these parameters and patient's clinicopathological features was analyzed statistically. In vitro, IL-8 concentration was evaluated in exogenous HGF-treated NPC cell lines by ELISA assay. The presence of EBV was also detected in NPC cells by PCR for Bam HI-W fragment. Both 54.3\% (69/127) cases of HGF high-expression in tumor cells and $80.3 \%(102 / 127)$ of HGF high-expression in stromal cells were significantly associated with increased microvessels density, advanced clinical stage, lymph node metastasis and high-expression of IL-8. Angiogenesis exhibited in relation to overall survival of NPC patients $(\mathrm{P}=0.001)$, and the patients with HGF and IL- 8 dual high-expression tumors had a significantly worse prognosis than those with single protein high-expression and dual low expression tumors ( $\mathrm{P}=0.011$ and $\mathrm{P}=0.026$, respectively). Exogenous HGF was observed to promote induction of IL-8 in NPC cells without EBV infection. Co-operating with IL-8, HGF might contribute to a poor prognosis of NPC by inducing angiogenesis through both autocrine and paracrine EBV-independent pathways.
\end{abstract}

\section{Introduction}

Nasopharyngeal carcinoma (NPC) is a malignant tumor that is well known to be associated with Epstein-Barr virus (EBV) and has a high incidence in southern China (1). NPC tumor cells are likely to invade to the surrounding tissue or metastasize to lymph nodes in the early stage of tumor

Correspondence to: Dr Zhi Li, Department of Pathology, 1st Affiliated Hospital, Sun Yat-sen University, 58 Zhongshan Road II, Guangzhou 510080, P.R. China

E-mail: lizhi@mail.sysu.edu.cn

Key words: angiogenesis, hepatocyte growth factor, interleukin 8, nasopharyngeal carcinoma, prognosis development, which is different from other head and neck cancers (2), and is the major cause for poor prognosis of this tumor. In clinical practice, the prognosis prediction of patients with NPC is mainly based on clinical TNM staging. However, it has been revealed that the TNM stage is sometimes inadequate to precisely predict patient prognosis because the NPC patients with the same TNM stage may frequently present different clinical outcome even if they are subjected to the same radiotherapy and adjuvant chemotherapy $(3,4)$. Whether or not certain molecules are involved in the development and progression of NPC, and consequently influence the prognosis of NPC is largely unknown.

The consistent presence of an inflammation-like microenvironment is a histopathological characteristic in primary tumor of NPC, especially in undifferentiated non-keratinizing carcinoma, which is the most frequent histological type of NPC in epidemic area. The recent data have suggested that a chronic inflammatory microenvironment not only facilitates the initiation of tumor, but also promote the growth, angiogenesis, invasion and metastasis of tumors through a complex network of inflammatory mediators, mainly produced by stromal cells $(5,6)$. Several cytokines and chemokines have been documented for their potent role in tumor progression, indicating an impact of these molecules on prognosis of tumors (7). Hepatocyte growth factor (HGF), a multifunctional cytokine induced by various types of cell, has various biological activities, which include mitogenic, morphogenic, motogenic, angiogenic and antiapoptotic effects (8). The activation of its high affinity receptor Met, has been demonstrated to promote tumor progression and metastasis, consequently inducing poor prognosis in numerous human cancers, including NPC (9). HGF is mainly produced by stromal cells in the tumor. Some studies have revealed that one of the important activities of HGF in promoting tumorigenesis and metastasis is to stimulate angiogenesis by a direct effect on endothelial growth and tube formation (10). However, whether or not stromal cells induce HGF to promote angiogenesis in NPC is still unknown, and the relationship between stroma-derived HGF itself and the prognosis of patients with NPC needs to be investigated.

Interleukin 8 (IL-8) is a prominent angiogenic molecule and has been proven to influence microvessel synthesis in 
various tumors (11). Angiogenesis is a key step in tumor growth, invasion and metastasis because massive formation of blood vessels in the tumor increases the opportunity for tumor cells to enter the circulation. Microvessel synthesis has been considered to influence tumor metastasis and prognosis in various human cancers (12). In NPC, several clinical studies, including our recent data, have demonstrated that intratumoral microvessel density is a strong independent predictor of poor prognosis of NPC (13-15). In vitro exposure of tumor cells to hypoxic conditions induces IL-8 expression by activating two transcription factors, activating protein-1 (AP-1) and nuclear factor-кB (NF-кB) $(16,17)$. In NPC, previous studies have focused on the effect of EBV infection and revealed that latent membrane protein 1 (LMP-1) can up-regulate IL-8 through an NF- $\mathrm{BB}$-mediated mechanism (18). EBV lytic transactivator Zta is also a potent inducer of IL-8, increasing IL-8 at both protein and RNA level and activating the IL-8 promoter (19). The relationship, if any, between HGF and production of IL- 8 by tumor cells in NPC needs to be elucidated. We investigated if HGF-induced IL-8 production in NPC can help clinicians improve the prognostic prediction and whether intervention of HGF in NPC could become a promising strategy for developing new therapeutic approaches to treat this malignant tumor.

The aim of current study was to evaluate whether HGF is a potent prediction factor for the prognosis of patients with NPC and whether stromal cells in tumor indeed possess tumor progression by inducing the HGF expression in NPC. We also assessed the correlation between HGF and IL-8 in their effect on angiogenesis and prognosis of 127 NPC patients. Our data suggest that both HGF autocrine and paracrine regulatory pathways, without co-operation of EBVencoded production, can independently promote tumor angiogenesis by inducing up-regulated expression of IL-8 in NPC. The co-elevated expression of HGF and IL-8 is a valuable prognostic marker for prognosis prediction in NPC patients.

\section{Materials and methods}

Specimens of NPC and clinicopathological findings. Archival formalin-fixed, paraffin-embedded specimens from 127 primary NPC patients before treatment during 1999-2005 in the 1st Affiliated Hospital, and Cancer Center of Sun Yat-sen University (Guangzhou, China) were recruited. The patients were 83 males and 44 females with a median age of 49 years (range 23-73). According to WHO histological classification, 109 patients were diagnosed as undifferentiated nonkeratinized carcinoma (UNKC) and 18 were diagnosed as differentiated non-keratinized carcinoma (DNKC). The patients were classified into the disease stage according to the criteria of China NPC 92 staging system (20). There were 9 in stage I, 33 in stage II, 50 in stage III and 35 in stage IV. All the patients were treated with standard curative radiotherapy with or without chemotherapy.

Immunohistochemistry and scoring. The sections were subjected to immunostaining using a ChemMate Envision/ HRP kit (Dako Co., Denmark). Slides were deparaffinized in xylene and rehydrated in decreasing concentrations of ethanol and rinsed in phosphate-buffered saline. The slides were incubated with hydrogen peroxide for $10 \mathrm{~min}$ following microwave with $10 \mathrm{mM}$ citrate buffer (pH 6.0; Sigma-Aldrich, Germany) at 5-min intervals for a total of $10 \mathrm{~min}$. After blocking with normal serum for $10 \mathrm{~min}$, the slides were incubated with a 1:100 dilution of rabbit polyclonal HGF antibody (Santa Cruz Biotechnology, USA), a 1:50 dilution of the mouse anti-human monoclonal IL-8 antibody (R\&D Co., USA), or a 1:100 dilution of mouse anti-human monoclonal CD34 antibody (Santa Cruz Biotechnology) for $60 \mathrm{~min}$, respectively. Slides were detected by ChemMate Envision/ HRP Kit for $30 \mathrm{~min}$ at room temperature and followed by developing with diaminobenzidine (DAB) for visualization. Negative controls were carried out by substituting nonimmune serum for the primary antibodies.

The immunostaining results were evaluated and scored independently by two pathologists without knowledge of the clinical data of patients. Evaluation of the immunostaining results was performed as previously described (21). HGF and IL-8 staining results were scored according to the percentage of cytoplasmic positive cells as follows: $(-),<10 \%$; (+), 11$20 \%$; (++), 21-50\%; (+++), $>50 \%$. The HGF immunostaining results were evaluated both in tumor cells and infiltrating lymphoid cells. The HGF high-expression was defined as $>20 \%$ in either tumor cells or stromal cells with positive staining, whereas HGF low-expression was $<20 \%$. IL- 8 highexpression was defined as $>10 \%$ tumor cells with positive staining, whereas IL-8 low-expression was $<10 \%$.

The counting of microvessels in NPC was evaluated by a previously reported method (22). Briefly, intratumoral microvessel density (IMD) was observed in areas of most intense neovascularization (hotspots) in tumor by light microscopy. After the area of highest neovascularization was determined, single microvessels were manually counted on a $200 \mathrm{x}$ field by two different observers without knowledge of patient outcome. Any brown-stained endothelial cell or cell cluster that was clearly separated from adjacent microvessels was considered as a single, countable microvessel.

Cell line and chemicals. In this study, the human NPC cell lines CNE-1 (a well differentiated NPC cell line), CNE-2 (a poorly differentiated NPC cell line), and lymphoblastoid cell lines, B95-8 (an EBV-infected lymphocyte cell line, as positive control for detecting EBV infection) were used. All three cell lines were maintained in RPMI-1640 culture medium with $10 \%$ heat-inactivated fetal bovine serum and antibiotics $(50 \mathrm{U} / \mathrm{ml}$ penicillin and $100 \mu \mathrm{g} / \mathrm{ml}$ streptomycin, Gibco/Invitrogen) at $37^{\circ} \mathrm{C}$ in a humidified incubator with $5 \%$ $\mathrm{CO}_{2}$. Human recombined hepatocyte growth factor (HGF), purchased from Sigma Chemical, was dissolved in culture medium for in vitro experiments.

Enzyme-linked immunosorbent assay (ELISA). The concentration of IL-8 was determined by ELISA kits (R\&D Systems, Inc.). CNE-1 and CNE-2 NPC cell lines were grown overnight in complete media at $5 \times 10^{4}$ cells in $1.0 \mathrm{ml} /$ well in sterile 24-well culture plates. The next day, two cells were switched to RPMI-1640 medium with $1 \%$ fetal bovine serum and treated with different doses of human recombinant HGF for $24 \mathrm{~h}$. Culture supernatants were collected and centrifuged 


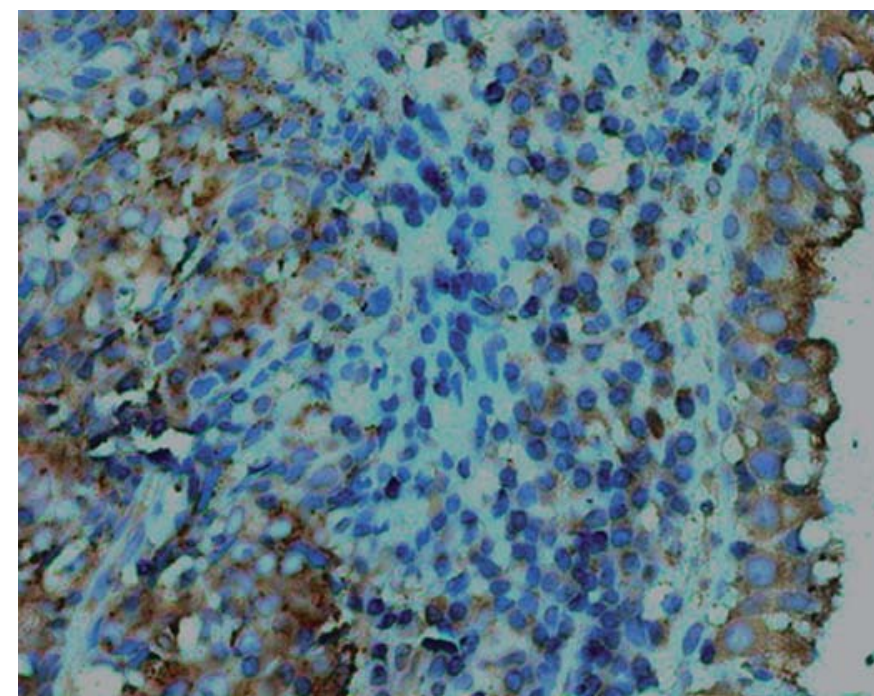

A

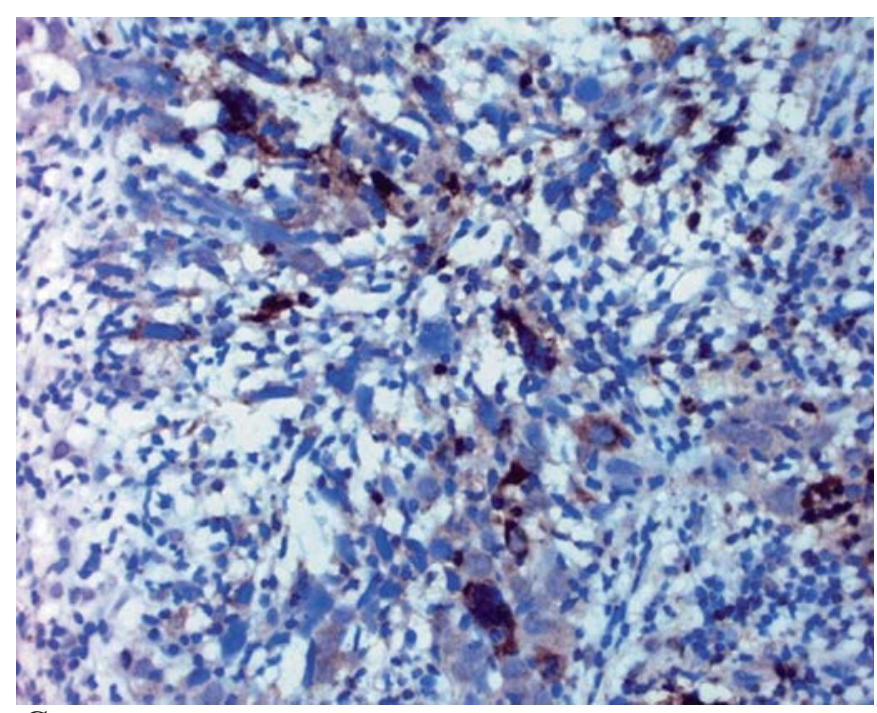

C
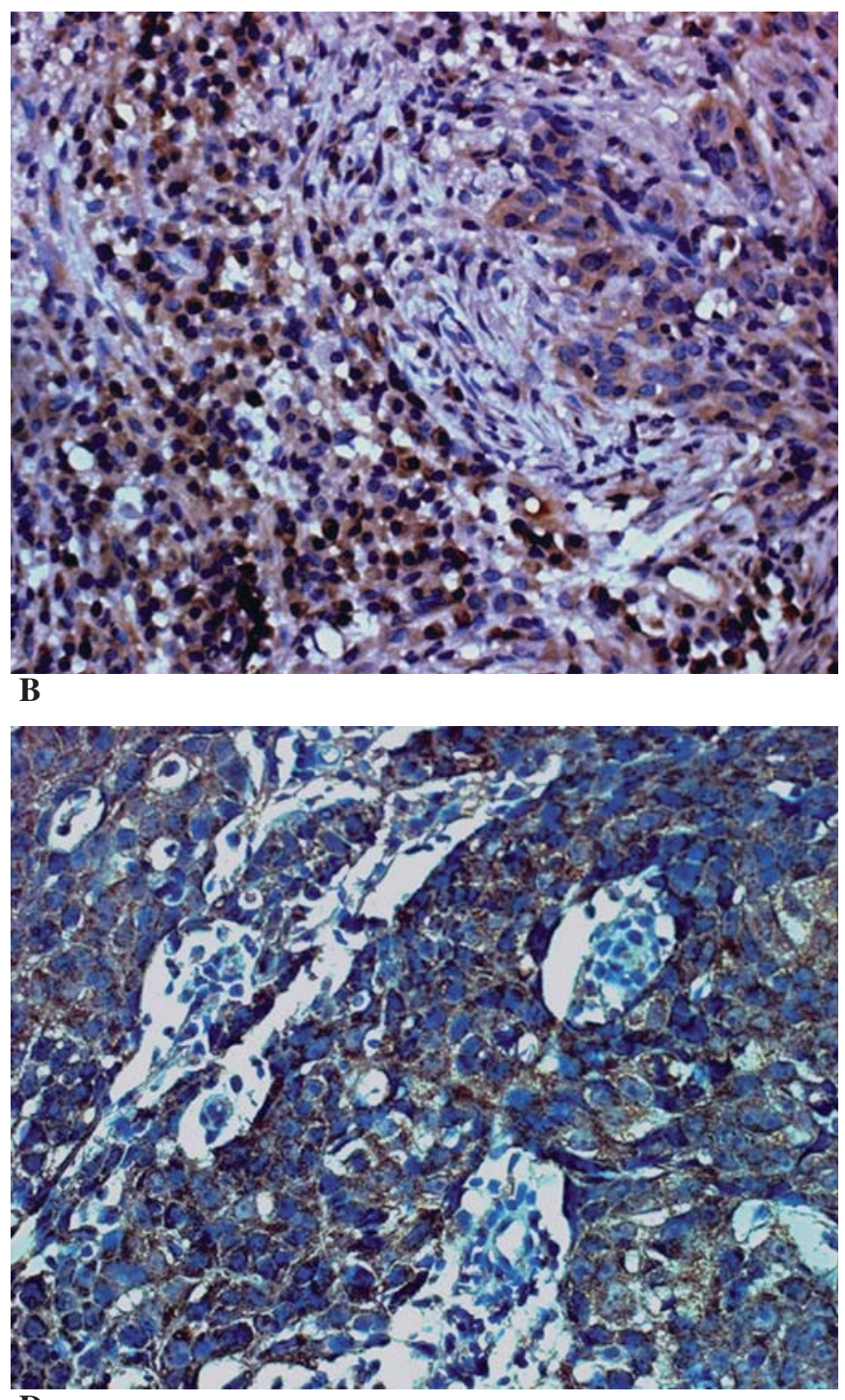

D

Figure 1. HGF and IL-8 expression in NPC patient biopsies. (A) HGF immunoreactivity was detected in membranous and cytoplasma of NPC tumor cells, stroma cells and normal adjacent lining epithelium of nasopharynx. (B) In some cases, strongly positive staining was observed of HGF either in stromal cells, including fibroblast and lymphoid cells, or tumor cells. (C) IL-8 positive immunostaining signal was localized on the cell surface and in the cytoplasm of tumor cells with scattered distribution pattern in most of cases. (D) in few cases, IL-8 protein was diffusely detected throughout the tumor cells. [(A) and (B) with original magnification $\mathrm{x} 200$; (C) and (D) with original magnification $\mathrm{x} 400$, immunohistochemical staining].

at $14000 \mathrm{rpm}$ for $5 \mathrm{~min}$ at $4^{\circ} \mathrm{C}$ to remove debris. Before the cytokine assay, $150 \mu 1$ of assay diluent was added to equilibrate the wells that had been precoated with monoclonal antibody specific to IL- 8 prior to the addition of $50 \mu 1$ of cell culture supernatant. The process of measurement was according to the manufacturer's instructions. The chromogenic reaction was identified by reading the absorbance at OD450 nm in a microplate autoreader. Each sample was tested in triplicate, and experiments were performed three times. The concentration of IL- 8 was interpolated from a standard curve performed by using recombinant cytokines, and expressed as $\mathrm{pg} / \mathrm{ml}$ of cell culture supernatant.

$D N A$ isolation and polymerase chain reaction $(P C R)$ for the detection of EBV infection. In this study, the determination of EBV infection in NPC cell lines was PCR method using primers to detect the Bam HI-W fragment of EBV as pervious description (23). Briefly, CNE-1, CNE-2 and B95-8 cell lines were cultured routinely and genomic DNA was isolated using TIANamp Genomic DNA Kit (Tiangen Biotech, Beijing, China). Template DNA $(0.1 \mu \mathrm{g})$ was added to $50 \mu 1$ reaction mixture containing polymerase chain reaction buffer, dNTP mixture, primers for EBV Bam HI-W (5'-ctttagaggcgaatggg cgc-3' and 5'-aggaccactttataccaggg-3'), and $5 \mathrm{U}$ Taq polymerase. PCR was performed in an automatic thermal cycler (Perkin-Elmer-Cetus, Norwalk, CT). Samples were amplified through 30 consecutive cycles with annealing temperature of $45^{\circ} \mathrm{C}$. A $10-\mu 1$ volume of each PCR product was analyzed by electrophoresis on $1.2 \%$ agarose gel containing $0.5 \mu \mathrm{g} / \mathrm{ml}$ ethidium bromide and the bands were visualized under ultra violet light. B95-8 cell line was used as EBV-positive control, and in negative control, DNA was replaced by TrisHCL/EDTA (TE) buffer.

Statistical analysis. All statistical analysis was carried out by SPSS 13.0 software for Windows. $\chi^{2}$ test was used to assess 
Table I. Correlation between proteins expression and clinicopathological parameters of NPC patients $\left(\chi^{2}\right.$ test).

\begin{tabular}{|c|c|c|c|c|c|c|c|c|c|c|}
\hline & \multirow[b]{2}{*}{$\begin{array}{c}\text { Case } \\
(\mathrm{n}=127)\end{array}$} & \multicolumn{3}{|c|}{ HGF in tumor cells } & \multicolumn{3}{|c|}{ HGF in stromal cells } & \multicolumn{3}{|c|}{ IL-8 } \\
\hline & & $\begin{array}{c}\text { High } \\
\text { expression } \\
(\mathrm{n}=69)\end{array}$ & $\begin{array}{c}\text { Low } \\
\text { expression } \\
(\mathrm{n}=58)\end{array}$ & P-value & $\begin{array}{c}\text { High } \\
\text { expression } \\
(\mathrm{n}=102)\end{array}$ & $\begin{array}{c}\text { Low } \\
\text { expression } \\
(\mathrm{n}=25)\end{array}$ & P-value & $\begin{array}{c}\text { High } \\
\text { expression } \\
(n=76)\end{array}$ & $\begin{array}{c}\text { Low } \\
\text { expression } \\
(n=51)\end{array}$ & P-value \\
\hline \multicolumn{11}{|l|}{ Age } \\
\hline$<49$ & 59 & 35 & 24 & 0.389 & 49 & 10 & 0.874 & 31 & 28 & 0.223 \\
\hline$\geq 49$ & 68 & 34 & 34 & & 53 & 15 & & 45 & 23 & \\
\hline \multicolumn{11}{|l|}{ Gender } \\
\hline Male & 83 & 41 & 42 & 0.158 & 66 & 17 & 0.875 & 54 & 29 & 0.162 \\
\hline Female & 44 & 28 & 16 & & 36 & 8 & & 22 & 22 & \\
\hline \multicolumn{11}{|l|}{$\begin{array}{l}\text { WHO histo- } \\
\text { type }\end{array}$} \\
\hline DNKC & 18 & 8 & 10 & 0.230 & 12 & 6 & 0.191 & 13 & 5 & 0.219 \\
\hline UNKC & 109 & 61 & 48 & & 90 & 19 & & 63 & 46 & \\
\hline \multicolumn{11}{|l|}{ T-stage } \\
\hline $\mathrm{T}_{1-2}$ & 52 & 28 & 24 & 0.924 & 37 & 15 & 0.203 & 36 & 16 & 0.147 \\
\hline $\mathrm{T}_{3-4}$ & 75 & 41 & 34 & & 65 & 10 & & 40 & 35 & \\
\hline \multicolumn{11}{|l|}{$\begin{array}{l}\text { Lymph node } \\
\text { status }\end{array}$} \\
\hline $\mathrm{N}_{0}$ & 34 & 12 & 22 & 0.008 & 16 & 18 & 0.001 & 16 & 18 & 0.089 \\
\hline $\mathrm{N}_{1-3}$ & 93 & 57 & 36 & & 86 & 7 & & 60 & 33 & \\
\hline \multicolumn{11}{|l|}{ Clincal stage } \\
\hline Stages I-II & 42 & 14 & 28 & 0.001 & 25 & 17 & 0.012 & 18 & 24 & 0.018 \\
\hline Stages III-IV & 85 & 55 & 30 & & 77 & 8 & & 58 & 27 & \\
\hline \multicolumn{11}{|l|}{ IL-8 expression } \\
\hline $\begin{array}{l}\text { Low } \\
\text { expression }\end{array}$ & 51 & 19 & 32 & 0.004 & 33 & 18 & 0.003 & & & \\
\hline $\begin{array}{l}\text { High } \\
\text { expression }\end{array}$ & 76 & 50 & 26 & & 69 & 7 & & & & \\
\hline
\end{tabular}

HGF and IL-8 expression with the clinicopathological characteristics. Non-parametric analysis of Mann-Whitney test was used to assess proteins expression in relation to angiogenesis of NPC. Results derived from in vitro study were given as the mean \pm SD as indicated. Data were analyzed by one-way ANOVA with Dunnett's post hoc test and Tukey's post hoc test for multigroup comparisons. The survival curve of patients was determined by Kaplan-Meier method and Cox regression, and statistical evaluation was performed using the log-rank test. $\mathrm{P}<0.05$ was considered statistically significant.

\section{Results}

$H G F$ and $I L-8$ expression in correlation to clinicopathological parameters in NPC. Immunostaining of HGF is presented in Fig. 1. Cytoplasmic positivity for HGF staining was observed either in tumor cells or in stromal cells, including fibroblast and lymphoid cells. The expression of HGF was also observed in the normal adjacent lining of epithelium of nasopharynx (Fig. 1A). Among 127 cases, we evaluated HGF expression either in tumor cells or stromal cells. For tumor cells, 25 (19.7\%) cases were negative (-), 33 $(25.9 \%)$ cases were $(+), 47(37.0 \%)$ cases were $(++)$, and 22 $(17.3 \%)$ were $(+++)$. High expression of HGF in tumor cells was observed in $69(54.3 \%)$ cases. In stromal cells, $4(3.1 \%)$ cases were negative $(-)$, whereas 123 cases were positive, of which $21(16.5 \%)$ were $(+), 56(44.1 \%)$ were $(++)$ and 46 $(36.2 \%)$ were $(+++)$. High expression of HGF in stromal cells of NPC was $80.3 \%$ (102/127) (Fig. 1B). High expression of HGF in either tumor cells or stromal cells was significantly associated with the advanced clinical stage (stages III and IV) and lymph node metastasis $(\mathrm{P}<0.05)$. However, no significant relationship between HGF high-expression and age, gender, histological type or T-stage was observed.

IL-8 positive immunostaining signal was localized on the cell surface and in the cytoplasm of tumor cells with scattered distribution pattern (Fig. 1C and D). Among 127 tumor tissues, IL-8 expression was negative (-) in 51 cases (40.2\%), whereas 49 cases $(38.6 \%)$ were $(+), 22$ cases $(17.3 \%)$ were $(++)$, and only 5 cases $(3.9 \%)$ were (+++). The high expression of IL-8 was observed in 76 cases $(59.8 \%)$. High expression of IL-8 


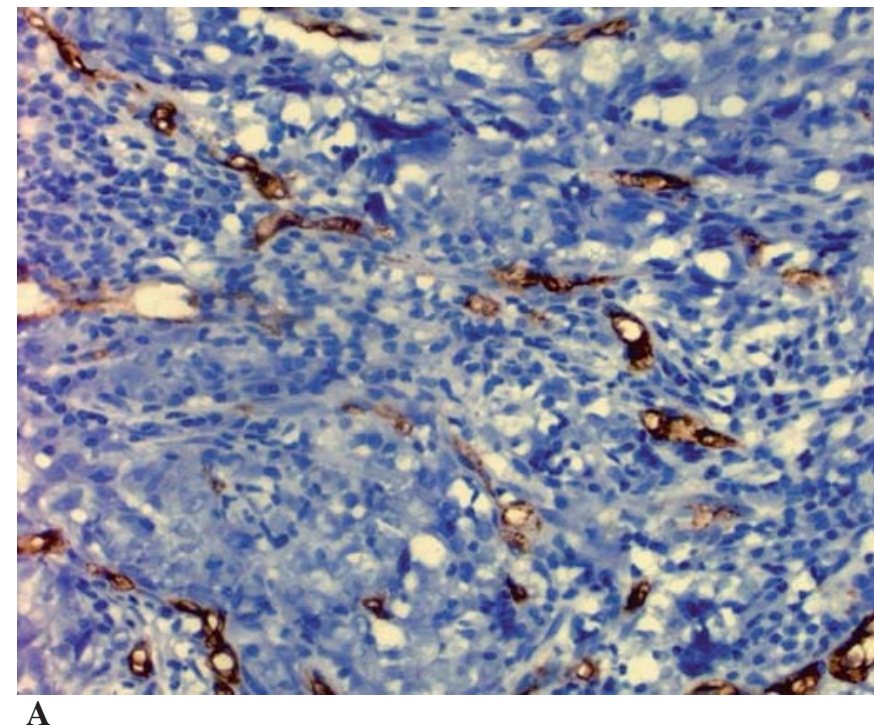

A

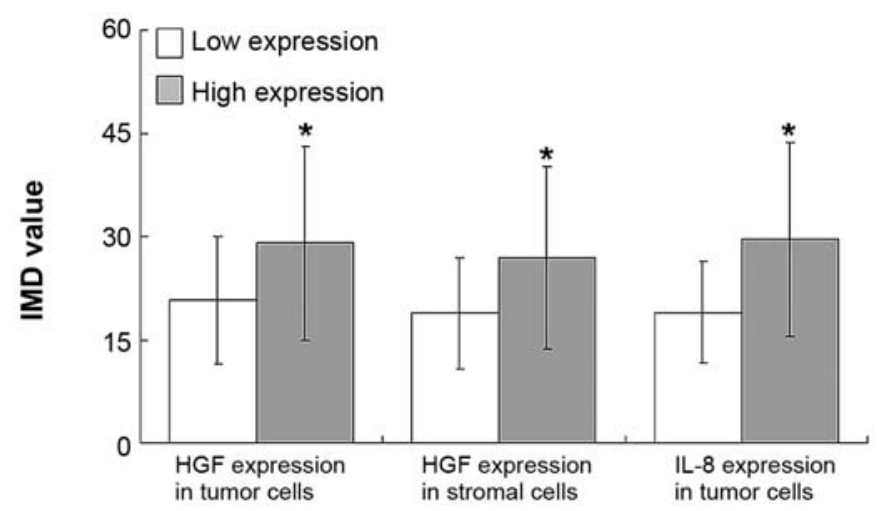

B

Proteins expression status in NPC

Figure 2. Intratumoral microvessels of NPC. (A) microvessels in tumor were highlighted by staining endothelial cells for anti-CD34. Any brown-staining endothelial cell or cell cluster that was clearly separated from adjacent microvessels was considered as a single, countable microvessel (with original magnification $\mathrm{x} 400$ ); (B) Correlation between intratumoral microvessel density (IMD) and protein expression in NPC, the mean IMD in the highexpressed HGF and IL- 8 cases was significantly increased compared to that of low-expressed cases $\left({ }^{*} \mathrm{P}<0.05\right)$.

was significantly associated with advanced clinical stage $(\mathrm{P}<0.05)$. There was no correlation observed between IL-8 expression and gender, age, tumor size and lymph node metastasis $(\mathrm{P}>0.05)$; but high expression of IL-8 exhibited a close correlation with high expression of HGF both in tumor cells and stromal cells of NPC (both P-values were <0.05) (Table I).

Relationship of HGF and IL-8 expression to microvessel counts. Microvessels in NPC, indicated by CD34 immunostaining, were detected as scattered in the tumor cell nests (Fig. 2A), and scored as intratumoral microvessel density (IMD). The correlation between IMD and proteins expression in NPC is shown in Fig. 2B. Mean IMD value was $29.06 \pm 14.17$ and $26.81 \pm 13.33$ in cases with HGF highly expressed in tumor cells and stromal cells, which were significantly higher than that of cases with HGF low expressed, respectively $(20.69 \pm 9.37$ and $18.82 \pm 8.02$, both P-values were $0.001)$. The microvessels were easier to observe in the 76 IL-8 high-expressed cases $(29.54 \pm 13.98)$ than those 51 tumors with IL-8 low expression $(18.84 \pm 7.36, \mathrm{P}=0.001)$. We examined the relationship between other clinicopathological variables and IMD in tumors; but no significant correlations were found. Therefore, angiogenesis in NPC appears to be independent of tumor size, stage, lymph node metastasis, histological type, sex and age of patients.

Association of HGF and IL-8 expression with survival of NPC patients. In this study, 61 cases had adequate follow-up data for the final analysis, whereas 66 cases were excluded from survival analysis because the patients were lost during the period of follow-up. The 61 cases were followed up from 2 to 53 months with mean period of 36 months, and 18 patients $(29.51 \%)$ had died of tumor in this period. The survival rates of patients with early clinical stage (stages I-II) and advanced stage (stages III-IV) were 77.27 and $66.67 \%$, respectively. There was no significant difference statistically $(\mathrm{P}=0.350$, Fig. 3A). The survival rate of NPC with IL- 8 high-expression or HGF high-expression in stromal cells was significantly lower than that in NPC patients with proteins low-expression, both $\mathrm{P}<0.05$ (Fig. 3B, C). The difference was not significant in survival rate between the NPC patients with different HGF expression status in tumor cells $(\mathrm{P}=0.069$, Fig. 3D). However, when the patients were stratified according to tumor high expression of both HGF and IL-8 in 61 cases, the survival rate of patients with dual high-expression of HGF and IL-8 in tumor cells was significantly lower than that of patients with dual low-expression or single protein high expression in tumor $(\mathrm{P}=0.004$, Fig. 3E). The patients with both highexpression of HGF in stromal cells and IL-8 high expression in tumor cells also obtained significantly longer survival than patients with dual low-expression or single protein high expression in NPC (P=0.011, Fig. 3F) (Table II).

Using Cox regression analysis of the entire group of patients, IMD was significantly associated with poor overall survival of NPC patients $(\mathrm{P}=0.001)$. High expression of HGF or IL-8 alone in NPC showed no significant correlation to the survival of patients. However, the patients with dual high expression of HGF and IL-8 in tumor cells, and the patients with both high expression of HGF in stromal cells and IL-8 had significant correlation to poor overall survival. Other clinical parameters, including age, gender, histological type, tumor size, lymph node metastasis and clinical stage, exhibited no association with the survival of patients (Table III).

Detection of EBV in NPC cells using the polymerase chain reaction method. Using polymerase chain reaction, the Bam HI-W fragment of EBV was detected in B95-8 cells. However, CNE-1 and CNE-2 cells showed no detectable EBV infection (Fig. 4).

Induction of IL-8 by exogenous HGF in NPC cells. The effect of exogenous HGF on IL-8 induction was evaluated by ELISA assay. NPC cell lines CNE-1, CNE-2 were exposed to $0-150 \mathrm{ng} / \mathrm{ml} \mathrm{HGF}$ for $24 \mathrm{~h}$, respectively. As shown in Fig. 5, exposure to HGF resulted in dose-dependent $(10-50 \mathrm{ng} / \mathrm{ml})$ enhancement of IL-8 concentration in culture supernatant. At the concentration of $50 \mathrm{ng} / \mathrm{ml}$, HGF exhibited its most 

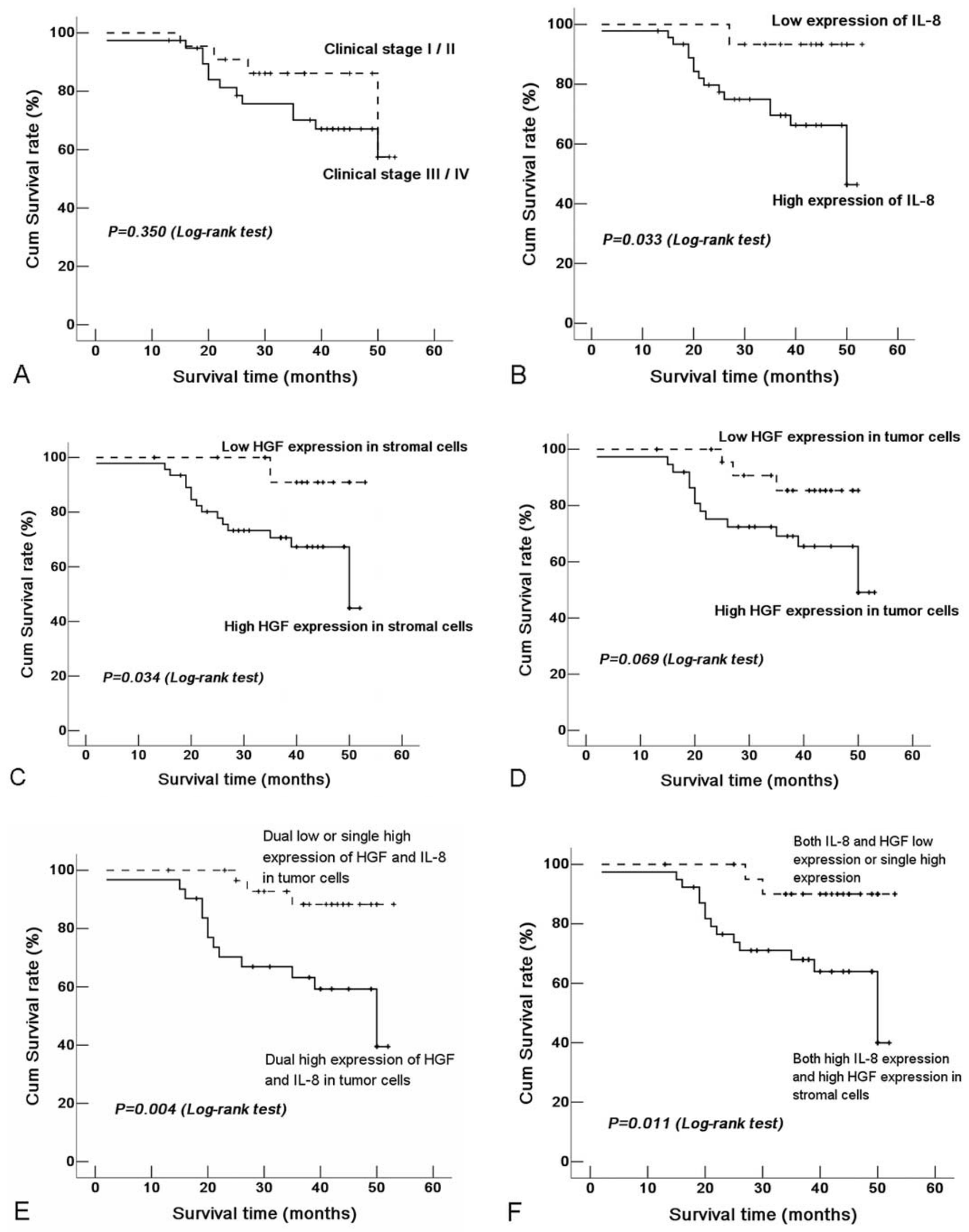

Figure 3. Kaplan-Meier survival analysis in 61 patients with NPC. (A) There was no significant difference in survival time between the patients in early clinical stage (I/II) and in advanced clinical stage (III/IV). Patients with IL-8 high-expressed tumor had a lower survival rate than those with IL-8 lowexpression (B). Patients with HGF high expression in stromal cells had a lower survival rate than those with low expression group (C); however, there was no significant difference between the patients with different HGF expression status in tumor cells (D). The patients with dual high expression of HGF and IL-8 in tumor cells exhibited remarkable decreased survival time compared to those with dual low or single protein high expression in tumor cells (E). The patients with both HGF high expression in stromal cells and IL-8 high expression had also a shorter survival (F). 
Table II. Kaplan-Meier analysis for overall survival rate of patients with NPC.

\begin{tabular}{lcc}
\hline Characteristics & $\begin{array}{c}\text { Mean survival } \\
\text { time (months) }\end{array}$ & $\begin{array}{c}\text { 95\% confidence } \\
\text { interval (months) }\end{array}$ \\
\hline Clinical stage & $47.71 \pm 2.37$ & $43-52$ \\
$\quad$ Early stage (I/II) & $42.43 \pm 2.36$ & $37-47$ \\
Advance stage (III/IV) & & $36-46$ \\
HGF in tumor cells & $41.76 \pm 2.59$ & $43-50$ \\
High expression & $46.96 \pm 1.64$ & \\
Low expression & & $37-46$ \\
HGF in stromal cells & $41.95 \pm 2.17$ & $48-54$ \\
High expression & $51.36 \pm 1.56$ & $37-46$ \\
Low expression & & $47-54$ \\
IL-8 expression & $41.94 \pm 2.19$ & \\
High expression & $51.26 \pm 1.67$ & $33-44$ \\
Low expression & & $47-53$ \\
HGF/IL-8 in tumor cells & $39.23 \pm 2.87$ & 0.034 \\
Dual high expression & $50.24 \pm 1.51$ & $36-45$ \\
Dual low or single high & & $47-53$ \\
HGF in stromal cells/IL-8 & $40.83 \pm 2.45$ & 0.033 \\
Both high expression & $50.56 \pm 1.64$ & 0.004 \\
Both low or single high & & \\
\hline
\end{tabular}

${ }^{\mathrm{a}} \log$-rank test.

Table III. Cox regression model correlating protein expression levels and clinicoparameters of NPC with overall survival of patients.

\begin{tabular}{lccr}
\hline Variables & Hazard ratio & $95.0 \%$ confidence interval & P-value \\
\hline Age & 0.502 & $0.194-1.297$ & 0.155 \\
Gender & 1.625 & $0.607-4.354$ & 0.334 \\
Histological type & 1.689 & $0.488-5.844$ & 0.408 \\
Tumor size & 0.444 & $0.166-1.188$ & 0.106 \\
Lymph node metastasis & 1.277 & $0.369-4.425$ & 0.699 \\
Clinical stage & 1.618 & $0.547-4.560$ & 0.363 \\
IMD & 1.097 & $1.059-1.137$ & 0.001 \\
HGF in tumor cells alone & 3.014 & $0.856-10.604$ & 0.086 \\
HGF in stromal cells alone & 6.455 & $0.858-48.542$ & 0.070 \\
IL-8 alone & 6.503 & $0.864-48.926$ & 0.069 \\
Dual high HGF/IL-8 in tumor cells & 5.056 & $1.425-17.60$ & 0.011 \\
High HGF in stromal cells and high & 5.316 & $1.221-23.144$ & 0.026 \\
IL-8 in tumor cells & & &
\end{tabular}

Result of Cox regression model analyzing age ( $<49$ vs. $\geq 49)$, gender (male vs. female), histological type (DNKC vs. UNKC), tumor size ( $T_{1-2}$ vs. $\mathrm{T}_{3-4}$ ), lymph node metastasis $\left(\mathrm{N}_{0}\right.$ vs. $\mathrm{N}_{1-3}$ ), clinical stage (I/II vs. III/IV), IMD (continuous variable), HGF in tumor cells (high vs. low expression), HGF in stromal cells (high vs. low expression), IL-8 (high vs. low expression), dual high HGF/IL-8 in tumor cells (dual high expression vs. dual low or single high expression), high HGF in stromal cells and high IL-8 (both high expression vs. dual low or single high expression).

effective induction of IL-8 either in CNE-1 or CNE-2 NPC cells. Compared with untreated cell, about 10- and 8-fold increase in IL- 8 secretion could be observed in CNE-1 and
CNE-2 cells, respectively. When the concentration was $>50 \mathrm{ng} / \mathrm{ml}$, the effect of HGF on promoting IL-8 secretion showed no significant difference compared to $50 \mathrm{ng} / \mathrm{ml}$. 


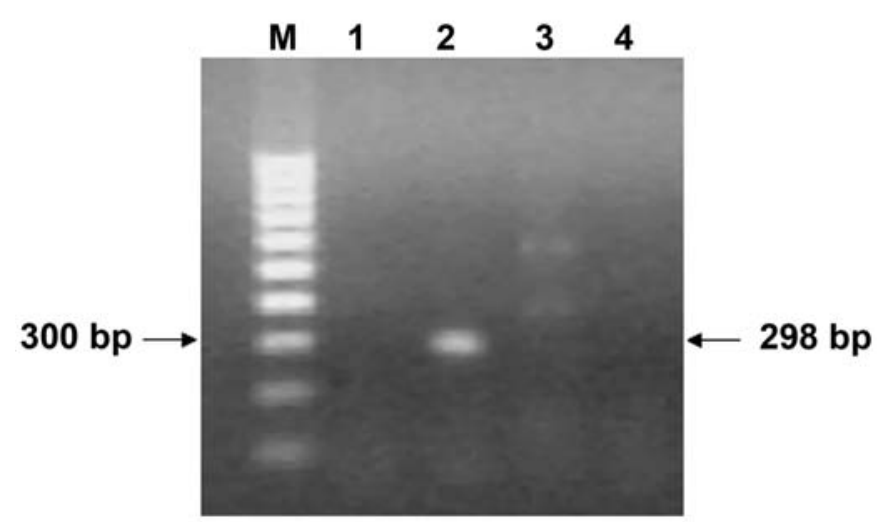

Figure 4. Presence of EBV infections in NPC cell lines using the polymerase chain reaction (PCR) method. Positive band represents the PCR product of EBV Bam HI-W fragment. Lanes: M, marker; 1, negative control; 2, B95-8 cell line (EBV positive control); 3, CNE-1 NPC cell line; 4, CNE-2 NPC cell line.

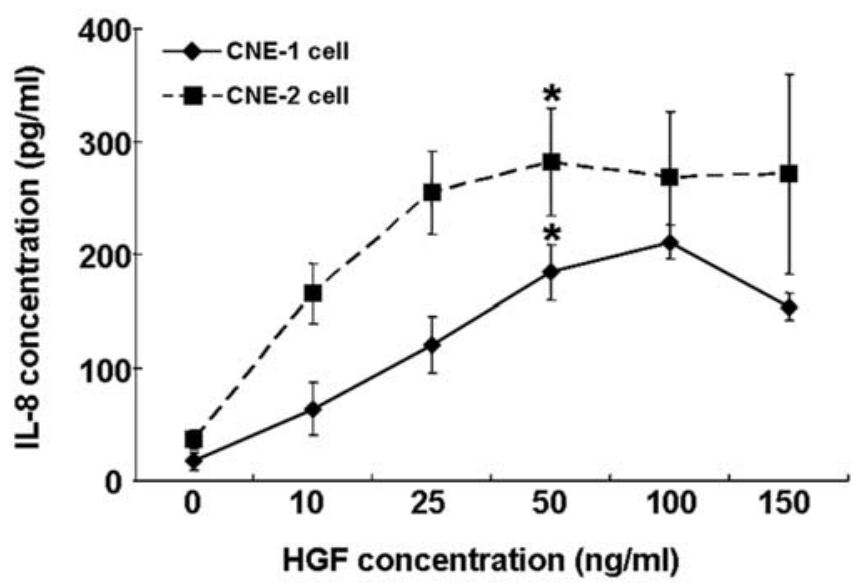

Figure 5. Induction of IL-8 in NPC cells by HGF. ELISA assay showed IL-8 concentration in culture supernatant varied in NPC cells with exogenous HGF treatment at various dosages. (Asterisk), a significantly increased IL-8 concentration was observed in NPC cells after 24-h treatment (one-way ANOVA test).

\section{Discussion}

The association of neovascularization with angiogenic and lymphatic metastasis has been investigated in many malignant tumors, and the contributions of angiogenic molecules such as VEGF, and IL-8 to the metastatic potential of tumors have been evaluated (24). But the effect of HGF on neovascularization and the relationship between HGF and IL-8 in NPC have not been identified. The present study suggested association of HGF and IL- 8 to neovascularization in NPC, and co-elevated expression of HGF and IL-8 in NPC was closely in correlation to poor prognosis of the patients. Although the number of NPC samples examined in this study was not sufficient to allow us to make definite conclusion, these results indicated that HGF may contribute to angiogenesis in NPC through the induction of IL-8. High expression of HGF may play important roles in NPC progression and influence the prognosis of NPC patients.
The importance of the HGF regulating system in cancer progression is still not fully understood. The present study shows that HGF may play a critical role in the mechanism of tumor metastasis and impact the clinical stage in NPC. High expression of HGF has been found in certain tumor cell lines derived from breast cancer and prostatic cancer, and HGFmediated cell migration and extracellular matrix invasion has been proven in a variety of normal and neoplastic epithelial cells in vitro $(25,26)$. Elevated serum HGF levels have also been detected to correlate with disease progression, with levels rising in cases of recurrence (27). In the current study, the result of relationship between HGF high expression and lymph node metastasis, as well as advanced clinical stage of tumor indicated that HGF might be a possible mediator contributing to the extensive lymph node metastasis and accelerating the poor progression of NPC. However, HGF high expression in tumor cells did not induce a poorer prognosis of patients independently, and significant shorted survival time was seen in patients whose tumor co-expressed HGF and IL-8. Moreover, HGF high expression in tumor cells was closely associated with higher microvessel density in tumor, which suggests tumor cell-derived HGF may contribute in part to angiogenesis of NPC. These results indicate that HGF high expression in NPC tumor cells might play an important role in lymph node metastasis through induction of angiogenesis, but it might not be an independent factor to influence the prognosis of NPC patients, although it specific receptor Met, has been regarded as a certain indicator to correlate with shorter survival in patients with late-stage NPC (9). Co-operating with other molecules, such as angiogenic molecules, high expression of HGF is a valuable prognostic marker for prognosis prediction in NPC. In contrast to our results, however, Yamashita et al (28) has reported that immunoreactive $\mathrm{HGF}$ is a strong and independent predictor of recurrence and survival in human breast cancer. Whether or not tumor species and sample size are responsible for these discrepancies require further investigation with larger cases and unabridged follow-up data needs to be clarified.

In the present study, we found that not only highexpressed HGF in tumor cells, but also high-expressed HGF in stromal cells were significantly correlated with lymph node metastasis, advanced clinical stage and high microvessels density in NPC tissues. Those results indicate that, besides autocrine regulatory loop, paracrine pathway of HGF may also influence tumor progression through induction of angiogenesis in NPC. In HGF-Met system, both pathways were important to execute their multifunctions. The co-expression of HGF and Met in tumor cells suggests the involvement of autocrine HGF-Met signaling loop. However, in the present study, only $54 \%$ cases showed high endogenous HGF in tumor cells, whereas $>80 \%$ cases had exogenous high HGF in stromal cells. Our previous study revealed that tumor cells in NPC had diffusely expressed Met protein (29). Collectively, we assume that in NPC, paracrine HGF-Met pathway might be more important to influence the tumor cell proliferation, migration and progression of NPC, although autocrine pathway might be also involved in these processes. It has been documented that paracrine interactions between stromal and cancer cells are crucial for determining the 
malignant behavior of a tumor (30), and induction of HGF by fibroblast directly promotes tumor cell invasiveness (31). Therefore, in NPC, it is reasonable to believe that HGF derived from stromal cells plays important roles in angiogenesis and progression of NPC by the paracrine pathway. Like tumor cell-derived HGF high-expression, stromal cell-derived HGF high-expression also failed to act as an independent factor for prognosis of NPC patients in survival analysis, although the patients with high expression of HGF in stromal cells had a significantly increased survival rate compared to those with low HGF expression; however, multivariate analysis indicated that both HGF high expression in stromal cells and IL-8 high expression in NPC was a strong independent predictor in NPC patients. That result suggests that co-analysis of HGF and IL- 8 protein expression in NPC tissue was valuable for prognosis evaluation of NPC patients.

In this study, angiogenesis in tumors was indeed independent of tumor size, lymph node metastasis and clinical stage in relation to the prognosis of NPC patient, similar to the findings of previous studies $(13,14)$. IL- 8 is considered to be one of the most prominent angiogenic molecules prompting tumor angiogenesis. Accumulated studies so far suggest that high expression of IL-8 has a positive influence on tumor growth, metastasis and progression $(32,33)$. In NPC, EBV induced IL-8 has been revealed to contribute in part to angiogenesis of tumors (18). We found that IL-8 high expression in NPC tumor cells was significantly correlated with advanced clinical stage and increased neovascularization in the tumor. These results provide additional evidence of the effect of IL-8 on tumor progression by promoting angiogenesis in the tumor. Multivariate analysis for overall survival in NPC patients indicated that IL-8 high expression in a tumor was not an independent prediction factor for prognosis of NPC although high expression of IL-8 was significantly correlated with decreased survival rate of NPC patients. IL-8 high expression in relation to high expression of HGF either in tumor cells or in stromal cells in NPC tissue, and exogenous HGF up-regulating IL-8 secretion in CNE-1 and CNE-2 NPC cells in vitro suggest the effect of HGF on induction of IL-8 in NPC. Previous studies have demonstrated that HGF can promote expression of IL-8 in head and neck squamous cell carcinoma and lung adenocarcinoma cells through activating MEK and PI3K signal transduction pathways $(34,35)$. However, before we confirmed the presence of EBV in NCP cells in our study, we could not determine whether or not the up-regulated expression of IL-8 was induced by EBV encoded protein or by exogenous HGF independently. Previous evidence has shown that IL-8 could be induced by EBV proteins, such as latent membrane protein 1 (LMP-1) through activating the NF- $\mathrm{BB}$ binding site (18), and lytic transactivator Zta through two Zta-responsive elements in the promoter of IL-8 (19). In the present study, we demonstrated that there was no EBV infection in two NPC cells used in our study. Exogenous HGF treatment alone induced up-regulation of IL-8 in vitro. These results suggest that HGF-induced IL-8 expression in NPC is independent of EBV. The precise mechanism involved is still to be investigated. Although tumor cells consistently harbor EBV DNA and some EBV encoded proteins in NPC tissues, we failed to demonstrate the relationship between LMP-1 expression and HGF or IL-8 expression in NPC tissues. The cooperative effect of HGF on IL-8 induction in EBV-infected NPC cells in vitro, might be different from that of HGF stimulation alone. Based on the above and previous results, we postulate that angiogenic promotion and regulation of IL8 expression in NPC may be a process including multiple inducing factors, such as EBV-encoded protein and HGF, as well as hypoxia involved in this process through their own specific pathways. HGF and IL-8 co-elevated tumor might be associated with worse prognosis in the patients with NPC; however, a single molecule expression status in tumor might not be a strong independent prognostic factor for NPC patients.

In conclusion, the present data provide a correlation among the HGF expression, IL-8 expression and survival of NPC patients. On the one hand, both HGF and IL-8 play an important role in contributing to angiogenesis in NPC, which is a consistently independent predictor for prognosis of NPC patients. On the other hand, neither HGF nor IL-8 alone is able to be an independent prognostic factor to predict accurately the patient prognosis although they indeed influence the lymph node metastasis and clinical stage. HGF might contribute to promote angiogenesis by inducing IL-8 expression through both autocrine and paracrine EBVindependent pathway. Therefore, co-elevated expression of HGF and IL-8 in NPC may be more valuable for prognosis evaluation of NPC patients. Currently, clinical stage alone is sometimes inadequate to precisely predict the patient prognosis, and the patients with the same TNM stage may frequently present different clinical outcomes. These two angiogenesis-related molecules could help clinicians improve the prognostic prediction. From this standpoint, diminishing the expression of HGF or interfering with the induction of IL-8 in tumor appears to be a promising strategy for developing new therapeutic approaches to treat this malignant tumor.

\section{Acknowledgements}

This study was supported by a grant from the Natural Science Foundation of Guangdong Province, China (5001744). The authors especially thank Dr Xiao Ying Tian, Hong Kong Baptist University, for her help in the preparation of the manuscript.

\section{References}

1. Yu MC and Yuan JM: Epidemiology of nasopharyngeal carcinoma. Semin Cancer Biol 12: 421-429, 2002.

2. Farias TP, Dias FL, Lima RA, Kligerman J, De Sa GM, Barbosa MM and Goncalves FB Jr: Prognostic factors and outcome for nasopharyngeal carcinoma. Arch Otolaryngol Head Neck Surg 129: 794-799, 2003.

3. Sakata K, Hareyama M, Tamakawa M, et al: Prognostic factors of nasopharynx tumors investigated by MR imaging and the value of MR imaging in the newly published TNM staging. Int J Radiat Oncol Biol Phys 43: 273-278, 1999.

4. Tatsumi-Tamori A, Yoshizaki T, Miwa T and Furukawa M: Clinical evaluation of staging system for nasopharyngeal carcinoma: comparison of fourth and fifth editions of UICC TNM classification. Ann Otol Rhinol Laryngol 109: 1125-1129, 2000.

5. Aggarwal BB, Shishodia S, Sandur SK, Pandey MK and Sethi G: Inflammation and cancer: how hot is the link? Biochem Pharmacol 72: 1605-1621, 2006. 
6. Lu H, Ouyang W and Huang C: Inflammation, a key event in cancer development. Mol Cancer Res 4: 221-233, 2006.

7. Balkwill F: Cancer and the chemokine network. Nat Rev Cancer 4: 540-550, 2004.

8. Boros P and Miller CM: Hepatocyte growth factor: a multifunctional cytokine. Lancet 345: 293-295, 1995.

9. Qian CN, Guo X, Cao B, et al: Met protein expression level correlates with survival in patients with late-stage nasopharyngeal carcinoma. Cancer Res 62: 589-596, 2002.

10. Grant DS, Kleinman HK, Goldberg ID, et al: Scatter factor induces blood vessel formation in vivo. Proc Natl Acad Sci USA 90: 1937-1941, 1993.

11. Waugh DJ and Wilson C: The interleukin-8 pathway in cancer. Clin Cancer Res 14: 6735-6741, 2008.

12. Thelen A, Scholz A, Benckert C, et al: Microvessel density correlates with lymph node metastases and prognosis in hilar cholangiocarcinoma. J Gastroenterol 43: 959-966, 2008.

13. Rubio L, Burgos JS, Lopez-Guerrero JA, Morera C and VeraSempere FJ: Expression of p53 protein and tumor angiogenesis as prognostic factors in nasopharyngeal carcinoma patients. Pathol Res Pract 198: 97-102, 2002.

14. Foote RL, Weidner N, Harris J, et al: Evaluation of tumor angiogenesis measured with microvessel density (MVD) as a prognostic indicator in nasopharyngeal carcinoma: results of RTOG 9505. Int J Radiat Oncol Biol Phys 61: 745-753, 2005.

15. Li Z, Bian LJ, Li Y, Liang YJ and Liang HZ: Expression of protease-activated receptor-2 (PAR-2) in patients with nasopharyngeal carcinoma: correlation with clinicopathological features and prognosis. Pathol Res Pract 205: 542-550, 2009.

16. Xu L, Pathak PS and Fukumura D: Hypoxia-induced activation of p38 mitogen-activated protein kinase and phosphatidylinositol 3-kinase signling pathways contributes to expression of interleukin 8 in human ovarian carcinoma cells. Clin Cancer Res 10: 701-707, 2004.

17. Maxwell PJ, Gallagher R, Seaton A, et al: HIF-1 and NF-kappaBmediated upregulation of CXCR 1 and CXCR2 expression promotes cell survival in hypoxic prostate cancer cells. Oncogene 26: 7333-7345, 2007.

18. Yoshizaki T, Horikawa T, Ren QC, et al: Induction of interleukin- 8 by Epstein-Barr virus latent membrane protein-1 and its correlation to angiogenesis in nasopharyngeal carcinoma. Clin Cancer Res 7: 1946-1951, 2001.

19. Hsu M, Wu SY, Chang SS, et al: Epstein-Barr virus lytic transactivator Zta enhances chemotactic activity through induction of interleukin-8 in nasopharyngeal carcinoma cells. J Virol 82: 3679-3688, 2008

20. Min H, Hong M, Ma J, et al: A new staging system for nasopharyngeal carcinoma in China. Int J Radiat Oncol Biol Phys 30: 1037-1042, 1994.

21. Nakashiro K, Hayashi Y and Oyasu R: Immunohistochemical expression of hepatocyte growth factor and c-Met/HGF receptor in benign and malignant human prostate tissue. Oncol Rep 10: 1149-1153, 2003.

22. Rubio L, Burgos JS, Morera C and Vera-Sempere FJ: Morphometric study of tumor angiogenesis as a new prognostic factor in nasopharyngeal carcinoma patients. Pathol Oncol Res 6: 210-216, 2000 .
23. Hirose Y, Masaki Y, Sasaki K, et al: Determination of EpsteinBarr virus association with B-cell lymphomas in Japan: study of 72 cases - in situ hybridization, polymerase chain reaction, immunohistochemical studies. Int J Hematol 67: 165-174, 1998.

24. Carmeliet P and Jain RK: Angiogenesis in cancer and other diseases. Nature 407: 249-257, 2000.

25. Zhou HY, Wan KF, Ip CK, Wong CK, Mak NK, Lo KW and Wong AS: Hepatocyte growth factor enhances proteolysis and invasiveness of human nasopharyngeal cancer cells through activation of PI3K and JNK. FEBS Lett 582: 3415-3422, 2008.

26. Spix JK, Chay EY, Block ER and Klarlund JK: Hepatocyte growth factor induces epithelial cell motility through transactivation of the epidermal growth factor receptor. Exp Cell Res 313: 3319-3325, 2007.

27. Fukuura T, Miki C, Inoue $\mathrm{T}$, Matsumoto $\mathrm{K}$ and Suzuki $\mathrm{H}$ : Serum hepatocyte growth factor as an index of disease status of patients with colorectal carcinoma. Br J Cancer 78: 454-459, 1998.

28. Yamashita J, Ogawa M, Yamashita S, Nomura K, Kuramoto M, Saishoji T and Shin S: Immunoreactive hepatocyte growth factor is a strong and independent predictor of recurrence and survival in human breast cancer. Cancer Res 54: 1630-1633, 1994.

29. Li Z, Lin SX, Liang HZ and He JH: Expression of hepatocyte growth factor/c-Met system in nasopharyngeal carcinoma and its biological significance. Zhonghua Bing Li Xue Za Zhi 34: $75-79,2005$

30. Kinzler KW and Vogelstein B: Landscaping the cancer terrain. Science 280: 1036-1037, 1998.

31. Kankuri E, Cholujova D, Comajova M, Vaheri A and Bizik J: Induction of hepatocyte growth factor/scatter factor by fibroblast clustering directly promotes tumor cell invasiveness. Cancer Res 65: 9914-9922, 2005.

32. Cacev T, Radosevic S, Krizanac S and Kapitanovic S: Influence of interleukin-8 and interleukin-10 on sporadic colon cancer development and progression. Carcinogenesis 29: 1572-1580, 2008.

33. Caruso DJ, Carmack AJ, Lokeshwar VB, Duncan RC, Soloway MS and Lokeshwar BL: Osteopontin and interleukin-8 expression is independently associated with prostate cancer recurrence. Clin Cancer Res 14: 4111-4118, 2008.

34. Dong G, Chen Z, Li ZY, Yeh NT, Bancroft CC and van Waes C: Hepatocyte growth factor/scatter factor-induced activation of MEK and PI3K signal pathways contributes to expression of proangiogenic cytokines interleukin- 8 and vascular endothelial growth factor in head and neck squamous cell carcinoma. Cancer Res 61: 5911-5918, 2001.

35. Chiang YY: Hepatocyte growth factor induces hypoxia-related interleukin-8 expression in lung adenocarcinoma cells. Mol Carcinog 48: 662-670, 2009. 